\title{
Childhood Obesity Interventions by Setting
}

\section{Tai Metzger ${ }^{1}$}

${ }^{1}$ National Cancer Institute, USA

\section{Correspondence}

Tai Metzger

Email: taimetzger@g.ucla.edu

\section{Publication Date}

January 04, 2021

MJM 2021 (19) 3

\section{(5) McGill \\ Journal of Medicine}

\author{
www.mjmmed.com
}

\section{cC) (1) (2) (2)}

This work is licensed under a Creative Commons BY-NC-SA 4.0 International License.

\section{ABSTRACT}

Background: To combat the global health crisis of obesity, many interventions have been implemented, including in children and adolescents. This age range is uniquely important because health behavior continues into adulthood, resulting in lifelong health risks or benefits. This narrative review aims to provide a cross section of the scientific literature regarding obesity interventions by setting, including schoolbased, daycare-based, home-based, healthcare-based, and digital-based, as well as to highlight gaps in research. Methods: Articles written in English addressing childhood and adolescent obesity interventions were sought online using PubMed and Google Scholar searches. Although some articles were from a global perspective, the majority focused on children in the United States. This search included reviews, individual studies, and other related papers. Results: School-based interventions are accessible to many, but there is limited evidence of long-term benefits. Home-based interventions were the only setting to have compelling evidence of long-term benefits, although there are several barriers to participation. Healthcare-based interventions are often successful when specific strategies and unique advantages of healthcare settings are utilized. Digital interventions have limited success now, but show potential for cost-effective scaling up as technology improves. Conclusion: The clearest gap in research is the lack of long-term studies, especially of school-based and healthcare-based interventions. Thus, it is imperative that investments are made into studies that include follow-up components continuing at least 1-2 years after the intervention. Additionally, home-based interventions have been more successful during early childhood while school-based interventions tend to be more successful during adolescence.

\section{KEYWORDS}

Obesity, childhood, obesity interventions, cancer prevention, adolescence 


\section{1 | INTRODUCTION}

Obesity is a growing public health crisis in North America and around the world. Childhood obesity prevalence, in particular, is alarmingly high, with $18.5 \%$ of children between the ages of two and 19 (13.7 million people) in the United States being classified as obese by the Centers for Disease Control and Prevention (CDC) in 20152016. (1) Since obesity is linked to hypertension, stroke, type 2 diabetes, coronary heart disease, and many other negative health outcomes, it is important to implement interventions to decrease obesity prevalence, starting in childhood. These negative health outcomes create massive costs for healthcare systems, so decreasing obesity rates would save much needed resources in addition to many lives. (2)

The CDC defines childhood overweight as having a body mass index (BMI) between the 85th and 95th percentile, and obesity over the 95th percentile for the same age and sex. Other methods of determining childhood obesity are also used, such as fat percentage of body weight, waist circumference, and skin fold thickness.

Childhood obesity interventions are generally implemented in one or more of these five settings: school, daycare, home and family (home and family will be used interchangeably throughout this paper), healthcare, and digital technology. The primary strategies of childhood obesity interventions are usually increasing physical activity (PA) and/or improving diet (e.g. decreasing caloric intake or replacing consumption of unhealthy foods with fruits and vegetables). While public policy interventions (e.g. soda taxes) also exist, this is not considered a setting for intervention for the purposes of this paper.

Schools are the most common setting for childhood obesity interventions, and have consequently been the subject of the most research. Thus, many different methods have been tried, some successful and some unsuccessful. Daycares have also been the setting for many interventions during early childhood. However, due to the COVID-19 pandemic, many classes and activities have been moved online and children spend much more time at home. (3) It is therefore more essential than ever to also understand childhood obesity interventions in the three other settings: the home, healthcare, and digital settings. This is illustrated in Figure 1.

As a major factor contributing to eating and activity behaviors of children, it is more important than ever for home and family life to be settings for treating and preventing childhood obesity. Interventions included in this category are programs that are carried out primarily at home. In this case, the parents are usually the ones who implement the program for their children. While many other settings often include parent involvement aspects, only interventions where the program is based almost entirely within the home will be included in this category.

The healthcare setting includes any interventions where the program is carried out through the healthcare system; a physician, nurse practitioner, physician assistant (PA), physical therapist, nurse, dietician, and/or other healthcare professional is the one who implements the program. Additionally, these interventions usually involve regular trips to the intervention site (usually a doctor's office or clinic) for monitoring adherence and outcomes, exercise training sessions, and/or diet and PA information sessions. While parents are often involved in healthcare-based interventions, if the primary implementer of the program is a healthcare professional or the program occurs primarily in a healthcare facility, it is categorized in the healthcare-based category. For example, if an intervention occurs primarily at a doctor's office, but includes help from the parents in implementing the program, then it is still considered healthcare-based.

The digital-based category is very broad. It encompasses any interventions where the primary mode of delivery is through technology, usually computers, cell phones, applications, and/or tablets. Like parents, technology is often a component of interventions based in other settings. However, only interventions where technology is the main (and often only) mode of delivery are included. While interventions based in the other settings sometimes include components to reduce screen time, digital-based interventions aim to use technology as a tool to reduce childhood obesity. 


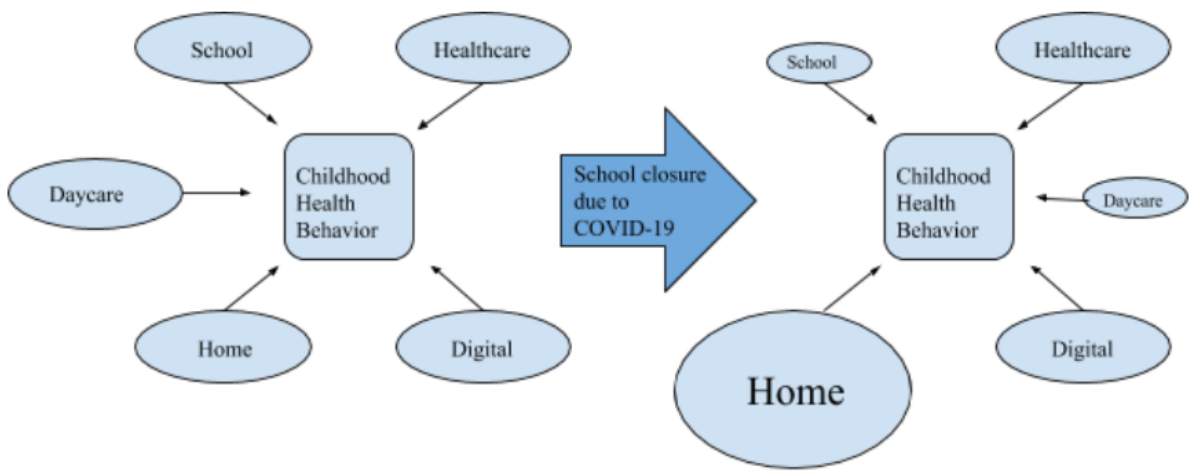

FIGURE 1 Due to COVID-19, classes have been moved online and many daycares have been closed, meaning that children are spending more time at home with their families. Consequently, Home and family-based obesity interventions must play a larger role in combating the obesity epidemic.

\section{2 | METHODS}

In this paper, interventions can target both overweight and obese children, and measurements other than BMI will also be considered. These interventions can be preventive or they can aim to decrease the weight in already obese or overweight children. Finally, "childhood" will be defined as anyone between the ages of zero and eighteen. Early childhood (from infancy to age 6), middle childhood (ages 7 to 11), and adolescence (ages 12 to 18 ) are the main subgroups for childhood in this paper. These age ranges correspond to the usual age at which children start first grade (age 7) and middle school (age 12). Using these criteria, Pubmed and Google Scholar were used to find studies, scientific reviews, and other relevant articles. Searches included the following phrases: "Childhood obesity interventions", "School-based childhood obesity interventions", "Daycare-based childhood obesity interventions", "Home-based childhood obesity interventions", "Family-based childhood obesity interventions", "Healthcare-based childhood obesity interventions", "Digital-based childhood obesity interventions", and "Technology-based childhood obesity interventions". Additionally, all these searches were repeated with "adolescent" substituted for "childhood." Over 150 papers were screened for relevance using their titles (or their abstracts in some cases, if the title was ambiguous). In total, 33 citations were used in this review. Only papers written in English were considered.

\section{3 | DISCUSSION}

\section{1 | School-based Interventions}

Many studies have found school-based interventions to be successful in the short-term. In a literature review of 20 interventions, primarily targeting early childhood, all interventions led to an improvement in at least one of their identified primary outcomes (e.g. BMI, incidence of overweight/obesity, PA, nutrition knowledge), although in some cases the improvements were very modest. (4) Key features identified by the authors as useful for improving results include incorporation of environmental factors and parent involvement. Environmental factors, which were more likely to be studied by international and more recent interventions, are factors that can hinder or enhance access to PA and/or healthy food. Parents have significant influence over their children's health behaviors and can be involved in a schoolbased obesity intervention through phone calls, meetings, or instructions to reinforce the information given at 
school. The authors also noted that each of the 20 interventions used different approaches to achieve success, suggesting that a "one-size-fits-all" approach is not necessary to produce positive results and that school-based interventions should utilize culturally specific strategies. (4)

The necessity of tailoring school-based interventions is evident in studies targeting the middle and, to a lesser extent, adolescent childhood age ranges as well. For example, an intervention to increase PA in low-income, urban middle schools used a curriculum provided by SPARK (Sports, Play, and Active Recreation for Kids) but did not result in an increase in moderate-to-vigorous physical activity (MVPA) which was the goal of the intervention. (5) The SPARK curriculum has been used in thousands of schools in the US and it is possible that the program was unsuccessful in this case because it was not modified sufficiently to meet the specific needs of low-income, urban schools. This need for modifications is supported by a previous SPARK intervention program which had no structured curriculum and resulted in increased MVPA. (6) In this intervention, Physical Education (PE) teachers were assisted in improving existing programs, instead of being given a specific program to follow. (6) Although this intervention may have been more successful because it was implemented at different schools (not low-income and urban), it could be that greater program flexibility played a part in the latter intervention's success.

Fewer studies specifically targeted high school students (adolescents). This lack of studies focusing specifically on adolescents is a problem because it is known that the shift from middle childhood to adolescence (and middle school to high school) is linked to a significant decrease in sports participation and PA more generally, as well as an increase in BMI. (7) One study of the "New Moves" intervention program targeting adolescent girls (8) included an all-girls physical education class ( $n=356$, $46 \%$ overweight or obese), supplemented with nutrition and self-empowerment components, individual sessions using motivational interviewing, lunch meetings, and parent outreach. Although no differences in weight status between the control (PE class did not use "New
Moves" curriculum) and intervention (PE class did use "New Moves" curriculum) groups were found, the program did result in improved sedentary activity rates, eating patterns, unhealthy weight control behaviors, and body/self-image. It is imperative that more interventions be developed that specifically target adolescents, especially interventions that are based in high-schools.

Some studies have explored interventions that target all three stages of childhood. One such intervention, which gave funding to schools in order to increase PA opportunities before, during, and after school, was implemented in 13 Colorado school districts. It successfully increased average daily PA from 48 minutes in the first year to 90 minutes by the end of the third year ( $p<$ .05), with schools reporting higher rates of free/reduced lunch students having less PA $(p=.04)$. Those involved in the intervention cited professional development and administrative support as reasons for its success. (9) Here, the exceptional support and training for the teachers who were implementing the program demonstrate the necessity of tailoring interventions to the needs of the school or school district. In this case, the intervention emphasized training and development which were desired by the schools.

According to a review of school-based obesity interventions for children and adolescents in the US and UK, (10) most of the examined interventions were based on a behavioral theory, usually social cognitive theory. This theory posits that parts of one's acquisition of knowledge comes directly from observing and interacting with others. (11) It makes sense that social cognitive theory would be the basis for school-based interventions because schools are a very social setting. However, this theory may not be as useful in other settings that do not involve as much interpersonal interaction, especially with peers of the same age range. This paper (10) concluded that school-based interventions should target both PA and nutrition behaviors, and that TV watching was the most modifiable behavior.

A clear limitation of school-based interventions is that even when positive results are found, improvements lasting more than a year after the program are rarely observed because of a lack of long term stud- 
ies. A 2008 review found that although many interventions have significant short-term improvements, very few have evidence of long-term effects. (12) The authors cite economic and environmental reasons as preventing long-term interventions and studies. Despite the high cost, research of this nature is necessary to determine the long-term effects of school-based childhood obesity interventions. Another important limiting factor is the difference in obesity rates among low socioeconomic status (SES) and high SES children. When designing interventions, researchers should consider the SES of the participants in order to better tailor the program to their specific needs.

\section{2 | Daycare-based}

Before children are old enough to start school, many of them spend much of their time at daycares (in 2012, $58 \%$ of children in the US ages 4 and 5 attended daycare). (13) This makes daycares a feasible setting for childhood obesity interventions that target early childhood. However, like high school-based interventions, few daycare-based interventions have been implemented. (14)

The Nutrition and Physical Activity Self-Assessment for Child Care (NAP SACC) developed a daycare-based intervention that involved dieticians with PA experience giving workshops, advice, and support regarding diet and PA to the daycare center staff, as well as educational information to the parents of the children. (14) In a study that aimed to determine the efficacy of this intervention, 26 daycare centers, with 209 total children, were split evenly into intervention and control groups (13 centers in each group). The intervention group increased total daily PA by 11.4 minutes ( $p<.05$ ), whereas the control group increased total daily PA by 2.5 minutes $(p<.05)$ after six months. (15) PA was tracked using accelerometers.

Prevention is also very important in the very early months of childhood. In a study examining an obesity prevention program in children under 2 years old ( $\mathrm{n}=$ 191), 3 intervention daycare centers ( 126 children) were compared with 3 control daycare centers (65 children).
(16) The intervention involved encouraging increased consumption of water, milk, fruits and vegetables, while increasing daily physical activity and decreasing daily consumption of sweets and savoury snacks and daily screen-time behavior. This was communicated through an informational poster and tailored feedback for parents about their child's PA and diet. However, over the course of a yearlong intervention, consumption of soft drinks and sweets increased while consumption of fruits and vegetables decreased. These findings highlight the importance of encouraging healthy behavior at a very early age. Although both the control and intervention groups experienced an increase in negative health behaviors, the intervention group had a significantly lower BMI. (16)

From these articles, (13-16) it is apparent that daycares are a necessary setting to promote healthy behavior during early childhood. Long-term studies should be conducted to test if these healthy behaviors carry on into the later stages of childhood and adulthood.

\section{3 | Home and Family-based}

As millions of children spend more time at home due to the current COVID-19 pandemic, it is essential to closely evaluate the efficacy and methods of homebased interventions. Decreases in PA during the quarantines and lockdowns could lead to permanently entrenched behavior in children. (17) While it is wellknown that parents influence the behavior of their children, it is important to establish the efficacy and duration of interventions implemented by parents to instill healthy behavior.

A 2011 meta-analysis of family-based childhood obesity interventions targeting all three stages of childhood found that the family is an effective setting for interventions. Family-based interventions produced significant weight loss in both the short-term and long-term. (18) Interestingly, the meta-analysis also suggested that the opposite sex parent plays a uniquely large role in influencing and sustaining a child's weight loss. In contrast, a 2014 review of family-based interventions in the UK (also of all three childhood age ranges) deter- 
mined that although short-term decreases in adiposity were evident, there was insufficient evidence to support long-term benefits. (19) This article did not rule out the possibility that family-based interventions can produce long term effects, but rather the authors did not find sufficient evidence of it in the UK. Thus, the 2011 article (18) is not contradicted by the 2014 one (19). The 2011 paper was likely able to find evidence of long term benefits because it was more comprehensive (a full meta-analysis of 20 studies was completed), whereas the 2014 article (19) only examined 10 studies, all of which were in the UK, and did not include a meta-analysis. Ultimately, home and family-based interventions can be effective for all three age ranges. In contrast to the very limited evidence of long-term benefits of school-based interventions, the literature regarding home-based provides some compelling evidence that home-based interventions have produced long-term results.

It is also important to determine what aspects of home and family activity are linked to decreased obesity. A very useful 2005 study focusing on preschoolers (early childhood) analyzed a nationally representative sample of 8,550 children to find associations between certain household routines and obesity prevalence. (20) The results were substantial: preschool-aged children who regularly ate dinner as a family, obtained adequate sleep, and had limited screen time had an approximately $40 \%$ lower prevalence of obesity compared to children who were exposed to none of these routines. Additionally, when analyzed individually, eating dinner as a family, obtaining adequate sleep, and having limited screen time were each linked to decreased prevalence of obesity by $4.4 \%, 3.9 \%$, and $3.9 \%$ respectively $(p<.005$ for each). A limitation of this study is that it is only correlational and did not involve an intervention, so a causal relationship cannot be established from these results. Although more recent data would be useful, it is apparent that these three home-based routines (regular family dinner, adequate sleep, and limited screen time) are connected to childhood obesity. Interventions targeting these routines are warranted, and interventions trying to find a causal relationship should target all three rou- tines for maximal effect.

One family-based intervention that targeted middle childhood attempted to decrease BMI in overweight and obese children (aged 9-12) by training and educating the parents and child about strategies to reduce screen time. (21) This study found no significant difference in BMI or screen time between the control and intervention groups, whereas the previous 2005 study found that children who had limited screen time were $4 \%$ less likely to be obese than those who did not. Several conclusions can be drawn from the difference in findings regarding the impact of reduced screen time between the previous study (20) and this one (21). Firstly, it could simply be that screen time and BMI have only a correlated relationship, not a causal one, since the 2005 study (20) was not an intervention. Another possibility is that a stated limit on screen time is more effective than educating parents and children about how to reduce screen time. In other words, being educated about strategies to reduce screen time may not have a significant effect on actual screen time. Finally, it is possible that household routines and habits are less impactful during middle childhood than in early childhood. The latter is supported by a systematic review comparing school-based and family-based interventions. (22) While both settings were found to have positive results, family-based interventions were found to be more effective for children under the age of 12 , and school-based interventions were more effective for children between the ages of 12 and 17. Since the children in the Maddison study (21) were between the ages of 9 and 12, it could be during this range (middle childhood) that the decrease in home-based efficacy begins to take effect.

While it is unclear why this difference in effectiveness exists, one study (23) found several ways that parent- and adolescent-reported barriers to intervention participation can be overcome. The most common barriers to participation reported by the adolescents included research demands (questionnaires, wearing accelerometers), program components (too much work, sessions were boring), and practical barriers (transportation, school work). On the other hand, the most common parent-reported barriers included program compo- 
nents (too many behavior changes, adolescent disapproval of being monitored), treatment motivation (lack of adolescent effort), parent-adolescent conflicts, and practical barriers. The authors state that parents and adolescents may find it easier to participate in interventions if "research and out?of?session program demands are minimized, efforts are made to enhance adolescent motivation, and treatment is offered in a convenient location and scheduled around school holidays and other family demands."(23, p1) Additionally, the results suggest that pre-emptively addressing adolescent unhappiness, family stressors, and parent-adolescent conflict could improve retention. These insights can be used to try to make family-based obesity interventions as successful during adolescence as they are earlier in childhood.

In summary, home-based obesity interventions can be effective on all childhood age ranges, although effectiveness decreases as children grow older (there is evidence (22) that there is an opposite trend in schoolbased interventions, however). Additionally, there is more evidence of long-term efficacy for home-based interventions than in school-based interventions. As children spend more time at home and less time at school due to the Covid-19 pandemic, home-based childhood obesity interventions, including preventive, have the opportunity to create lasting change.

\section{4 | Healthcare-based}

A 2015 review of primary-care based pediatric obesity interventions for children ages 0-18 compared 31 studies and identified eight interventions that had significant positive results. (24) The interventions were carried out by a physician, nurse practitioner, physician assistant, nurse, dietician/health coach, and/or a psychologist. All eight successful interventions had two things in common. First, they had parent-targeted components, although for the successful adolescent interventions parents had a more limited role. Second, they targeted multiple weight-related behaviors, and tended to use multiple modes of delivery. For example, one intervention (25) included "1) computer-guided behavior change plan and behavioral assessment for the adolescent; 2) inperson physician visit to discuss the adolescent's physical activity, nutrition, and sedentary behaviors and their behavior change plan; 3) adolescent and parent session with study PI [principal investigator] to learn food selfmonitoring; 4) adolescent phone coaching sessions with a study counselor; and 5) informational materials for the adolescent and parent." (24, p6) Other common, but not universal, components of the successful interventions were daily caloric goals/plans, daily PA goals/plans, and regular weighing.

Additionally, the authors cited several advantages to healthcare-based interventions over other settings. (24) First, they can build on the pre-existing relationships between physician, child, and family. Second, parents and children regularly go to the doctor's office together. Third, families generally trust the medical knowledge of healthcare professionals. However, the authors also cited some disadvantages associated with healthcarebased interventions. Time is often a constraint for families who do not want to drive to the doctor's office regularly. Additionally, some medical professionals believe parents are not always concerned with the weight of their child. (24)

The efficacy of healthcare-based interventions is also supported by a study of 100 overweight or obese children aged 5 to 14 who underwent an intervention with their family. (26) $13 \%$ of the children in the intervention group $(n=100)$ became normal weight, compared to $4 \%$ of the obese or overweight children in the control group ( $n=943$ ). (26) No dropouts were noted. This intervention involved many different health professionals; a physician, dietician, physical activity coach, and psychotherapist all had key roles in the intervention. Furthermore, this study is especially useful because it included follow-up research to determine the long-term effects of the intervention, which is rarely incorporated into childhood obesity intervention studies. In this case, the intervention group sustained improved weight compared to the control group for over 14 months when follow-up research ended. Although more studies are needed to confirm this long-term success, this study suggests that healthcare-based interventions can lead 
to enduring improvements.

Healthcare-based childhood obesity interventions have shown some positive results when they incorporate several key components, such as parent involvement, multiple modes of delivery, and multiple health professions. While some research has demonstrated long-term improvements, more studies with long term follow-up components are necessary to verify this.

\section{5 | Digital-based}

Because technology is improving so rapidly, it is important to identify how the digital setting can be harnessed to effectively develop childhood obesity interventions. Interestingly, nearly all research regarding digital-based interventions focuses on adolescents. Perhaps this is because reducing screen time is a major focus of childhood health interventions and digital obesity interventions may conflict with these goals, or because adolescents are very adept at using technology. Thus, not every technology platform had studies for each age group.

One study that did address early childhood utilized multiple digital-based methods for intervention. (27) The goal was to prevent childhood obesity in their first two years, and consisted of "scientifically substantiated content, tools, and telephone-based professional support delivered in an anticipatory and sequential manner via the internet, email, and text messages, focusing on educational modules addressing the modifiable factors associated with childhood obesity." (27, p1) This paper only outlined the design of the intervention, incorporating best practices, but did not address whether or not it resulted in decreased childhood obesity. However, the paper did conclude that a multi-component digital intervention is a cost-effective method that fits into the lifestyle of new mothers and has the potential to be scaled up to make real and sustainable change. These conclusions were based on the fact that the intervention design met criteria for intervention best practices, as well as followed theoretical and behavioral frameworks.

Virtual reality (VR) has also been used to increase childhood PA. One study examining "exergaming" (the use of VR and other digital video games to increase PA) during middle childhood, yielded several important insights. (28) Existing exergames such as "Wii Sports" and "Dance Dance Revolution" produced PA equivalent to a brisk walk and were therefore better than the sedentary behavior associated with traditional video games. However, according to several studies cited in the article, PA produced by exergaming is not vigorous enough to replace participation in sports, implying that exergames could be used to complement-rather than replace-sports participation. It is also expected that with continued development of VR technology, the intensity of exergames will be able to increase.

A major method for delivering digital obesity interventions to adolescents is texting, the preferred mode of communication for teenagers. (29) An intervention involving texts with goal prompts (e.g. texts to set step goal for the week) and Self Determination Theory-informed messages resulted in modest increases in steps per day and PA. (30) Such texts promoted the satisfaction of the three basic psychological needs-autonomy (having choices), competency (having skills and knowledge), and relatedness (having connections with oneself and others)-that are outlined by the theory. Not only did participating adolescents record increases in steps and PA (using pedometers), but they also reported positive reactions to the intervention. Another intervention for children (not just adolescents) involving texting used text-based healthcare chatbots (THCB) to have conversations about health behavior with the participants. (31) While only preliminary results have been published and no data regarding obesity reduction has been released, the preliminary findings suggest that the chatbots are effective in having multiple interactions with the participants and the program seems to be scalable for greater use.

Smartphone applications are also being explored as a potential mode of delivery for obesity interventions. A study of the efficacy of a nutrition education app for adolescents found that $76 \%$ of the participants preferred getting the information from the smartphone app rather than brochures. (32) This preference for digitalbased delivery of information can be applied to the other settings as well, since they often include an edu- 
cational component. Switching from brochures to apps could improve transmission of information, particularly with adolescents, but simply improving knowledge is not enough to guarantee behavioral change.

Considering that digital-based interventions are new and rapidly changing, it is important to keep in mind several important strategies when creating these kinds of interventions. A paper published in 2018 outlines three key practices for use in technology-based childhood obesity interventions. (33) These user-centered strategies are: co-designing with adolescents and children, personalization (developing individualized programs), and just-in-time adaptation (often using data from wearable devices).

Taken as a whole, texting, smartphone applications, exergaming and VR, and multi-component interventions show potential to cost-effectively improve childhood obesity on a large scale. However, most research regarding digital-based obesity interventions has focused on adolescents and more attention should be given to early and middle childhood. Additionally, more research should be done to explore the extent of the benefits of technology-based interventions (i.e. Does improved transmission of knowledge via digital devices lead to increased behavioral changes? How long do benefits last?).

\section{CONCLUSIONS}

As obesity continues to be a health crisis in North America and the world, it is imperative that effective childhood interventions be implemented. The overarching goal of childhood obesity interventions is to prevent the long-term negative health consequences of obesity from carrying into the adulthood of yet another generation. Unfortunately, the COVID-19 pandemic has distorted the daily lives of children and parents across the planet, changing how and where our time is spent. Thus, it is essential to understand the different settings for childhood obesity interventions and what strategies work best in each setting.

Several important conclusions can be drawn from the current literature regarding school-based interventions. Overall, school-based interventions do show signs of effectively producing short-term improvements in obesity, but more studies are needed to determine if there are long-term benefits. Additionally, when implementing obesity interventions at schools, a "one-size-fits-all" approach may not be as effective as tailoring each intervention to the specific needs of each school or school district. Additionally, because participation in structured physical activities, particularly organized sports, among children decreases from middle school to high school, and because school-based interventions appear to become relatively more effective than home-based interventions as age increases, more studies should be done that focus directly on high school interventions.

Daycares, which can be a setting for intervention during early childhood, have been the site of multiple successful programs. The NAP SACC intervention was particularly effective at increasing MVPA. Additionally, it is important for daycare-based interventions to pay close attention to diet during early childhood, which becomes significantly unhealthier over a child's first 2 years. Interventions during early childhood can build a firm foundation of healthy behavior that can hopefully carry on into middle childhood, adolescence, and adulthood.

With the closure of many schools and daycares during the pandemic, childcare is taking place more frequently within homes. Families, therefore, will likely influence their children's health behavior even more than is already known. Studies of home-based interventions have found significant short-term, and in some cases long-term, decreases in obesity. Additionally, several household family routines have been linked to decreased prevalence of childhood obesity (eating dinner as a family, limited screen time, and adequate sleep), and interventions should explore implementing all three of these. Another key finding has been the relationship between the impact of setting on the efficacy of an intervention and the child's age: according to some evidence, home-based interventions are more impactful with younger children (0-12) while school-based interventions are more impactful with older children (12-18). Overall, family-based interventions currently seem to 
be the most effective because of the potential for longterm benefits. However, the other settings have advantages as well.

Healthcare has also been assessed as a setting for childhood obesity intervention because families make regular visits and because they usually trust the information given by physicians and other healthcare professionals. Successful healthcare-based interventions tend to involve multiple modes of delivery (meetings with physicians, computer assessments, phone calls with dieticians) and parent involvement. Instances of longterm impacts have been found, but more studies are necessary to confirm this.

Finally, digital-based interventions, utilizing various types of technologies, have shown potential to be effective during childhood, with adolescents almost always being the focus of studies. Texting and smartphone applications have been effectively used with adolescents to increase MVPA and improve nutrition knowledge, respectively. Other strategies and technologies that may improve efficacy are personalization, co-designing interventions with children and adolescents, just-in-time adaptation (usually with wearable devices such as accelerometers or pedometers), and exergames (improvements in exergaming, especially with VR, can increase intensity). Digital-based interventions are especially exciting because of their potential for cost-effective scaling up as technology improves.

Overall, the single most important conclusion that can be drawn from the literature is the need for research into the long-term effects of these interventions. This means studies that continue for years in order to determine which interventions lead to lasting benefits. While the cost of such research is extremely high, the benefits of combating the obesity pandemic outweigh these costs.

\section{1 | Future Research Questions}

The most notable research gap is the lack of sufficient follow-up components of studies to determine if the effects of interventions continue in the years following the intervention. These are especially needed for school-based and healthcare-based interventions. Several other questions for future research include: What are the effects of school-based obesity interventions that directly target high school students? How can family-based interventions be improved and adjusted in response to the increase in time spent at home during the pandemic? Can digital-based interventions be effective during early and middle childhood? How can digital-based interventions be cost-effectively scaled up to make significant global change? How can the SES obesity gap be effectively addressed by childhood obesity interventions?

\section{References}

1. Hales CM, Carroll MD, Fryar CD, Ogden CL. Prevalence of Obesity Among Adults and Youth: United States, 20152016. National Center for Health Statistics Data Brief [Internet]. 2017 October [cited 2020 Aug 25]; 288. Available from: https://www.cdc.gov/nchs/data/databriefs/db288.pdf

2. Wharton S, Lau DCW, Vallis M, Sharma AM, Biertho L, et al. Obesity in adults: a clinical practice guideline. Can Med Assn J [Internet]. 2020 Aug [cited 2020 Nov 7]; 192(31): E875-891. Available from: https://www.cmaj.ca/content/cmaj/192/31/E875.full.pdf

3. Power K. The COVID-19 pandemic has increased the care burden of women and families. Sust: Sci Prac Pol [Internet]. 2020 Jun [cited 2020 Nov 7]; 16(1): 67-73. Available from Taylor \& Francis Online: https://www.tandfonline.com/doi/full/ 10.1080/15487733.2020.1 776561

4. Ickes JM, McMullen J, Haider T, Sharma M. Global School-Based Childhood Obesity Interventions: A Review. Int J Environ Res Public Health [Internet]. 2014 Aug [cited 2020 Aug 25]; 11(9): 8940-8961. Available from PMC: https://www.ncbi.nlm.nih.gov/ pmc/articles/PMC4198999/

5. Gill M, Roth SE, Chan-Golsten AM, Rice LN, Crespi CM, KoniakGriffin D, et al. Evaluation of an Intervention to Increase Physical Activity in Low-Income, Urban Middle Schools. J Sch Health [Internet]. 2019 Jul [cited 2020 Aug 25]; 89(9): 705-714. Available from Wiley: https://onlinelibrary.wiley.com/doi/epdf/10.1111/josh.12808 6. McKenzie TL, Sallis JF, Prochaska JJ, Conway TL, Marshall SJ, et al. Evaluation of a two-year middle-school physical education intervention: M-SPAN. Med Sci Sports Exerc [Internet]. 2004 Aug [cited 2020 Aug 25]; 36(8): 1382-1388. Available from: https://pdfs.semanticscholar.org/8a04/96ed5f9c46014358168b8 fca02665981f4ca.pdf

7. Turner RW, Perrin EM, Coyne-Beasley T, Peterson CJ, Skinner AC. Reported Sports Participation, Race, Sex, Ethnicity, and Obesity in US Adolescents From NHANES Physical Activity (PAQ_D). Glob Pediatr Health [Inter- 
net]. 2015 Apr [cited 2020 Aug 25]. Available from PMC: https://www.ncbi.nlm.nih.gov/pmc/articles/PMC4784630/

8. Neumark-Sztainer DR, Friend SE, Flattum CF, Hannan PJ, Story MT, et al. New moves-preventing weight-related problems adolescent girls a group-randomized study. Am J Prev Med [Internet]. 2010 Nov [cited 2020 Aug 25]; 39(5): 421-432. Available from PMC: https://www.ncbi.nlm.nih.gov/pmc/articles/PMC2978965/

9. Kelly C, Carpenter D, Behrens TK, Field J, Luna C, et al. Increasing Physical Activity in Schools: Strategies for School Health Practitioners. Health Promot Pract [Internet]. 2019 June [cited 2020 Aug 25]; 20(5): 607-702. Available from PMC: https://pubmed.ncbi.nlm.nih.gov/31238744/

10. Sharma M. School-based interventions for childhood and adolescent obesity. Obesity Reviews [Internet]. 2006 Aug [cited 2020 Aug 25]; 7(3): 261-269. Available from PMC: https://pubmed.ncbi.nlm.nih.gov/16866974/

11. Bandura A. National Inst of Mental Health. (1986). Prentice-Hall series in social learning theory [Internet]. Social foundations of thought and action: A social cognitive theory. Prentice-Hall, Inc [cited 2020 Aug 25]. Available from: https://psycnet.apa.org/record/1985-98423-000

12. Shaya FT, Flores D, Gbarayor CM, Wang J. School-based Obesity Interventions: A Literature Review. J School Health [Internet]. 2008 March [cited 2020 Aug 25[; 78(4): 189-196. Available from PMC: https://pubmed.ncbi.nlm.nih.gov/18336677/

13. Rathbun A, Zhang A, Snyder TD. Primary Early Care and Education Arrangements and Achievement at Kindergarten Entry. National Center for Education Statistics [Internet]. 2016 Jun [cited $2020 \mathrm{Nov}$ 7]. Available from: https://nces.ed.gov/pubs2016/2016070.pdf

14. Ammerman AS, Ward DS, Benjamin SE, Ball SC, Sommers JK, et al. An Intervention to Promote Healthy Weight: Nutrition and Physical Activity Self-Assessment for Child Care (NAP SACC) Theory and Design. Prev Chronic Dis [Internet]. 2007 July [cited 2020 Aug 25]; 4(3). Available from: https://www.cdc.gov/pcd/issues/2007/jul/06_0115.htm

15. Bonis M, Loftin M, Ward D, Tseng TS, Clesi A, et al. Improving Physical Activity in Daycare Interventions. Childhood Obes [Internet]. 2014 Aug [cited 2020 Aug 25]; 10(4). Available from: https://www.liebertpub.com/doi/full/10.1089/chi.2014.0040

16. Verbestel V, Coen VD, Winckel MV, Huybrechts I, Maes L, et al. Prevention of overweight in children younger than 2 years old: a pilot cluster-randomized controlled trial. Pub Health Nutr [Internet]. 2013 May [cited 2020 Aug 25]; 17(6): 1384-1392. Available from PMC: https://pubmed.ncbi.nlm.nih.gov/23701835/

17. Dunton GF, Do B, Want SD. Early effects of the COVID19 pandemic on physical activity and sedentary behavior in children living in the US. BMC Pub Health [Internet]. 2020 Sept [cited 2020 Nov 7]; 20(1): 1351. Available from PMC: https://www.ncbi.nlm.nih.gov/pmc/articles/PMC7472405/

18. Berge JM, Everts JC. Family-Based Interventions Targeting
Childhood Obesity: A Meta-Analysis. Childhood Obes [Internet]. 2015 July [cited 2020 Aug 25]; 7(2). Available from PMC: https://www.ncbi.nlm.nih.gov/pmc/articles/PMC4504253/

19. Upton P, Taylor C, Erol R, Upton D. Family-based childhood obesity interventions in the UK: a systematic review of published studies. Community Practitioner [Internet]. 2014 July [cited 2020 Aug 25]; 87(5): 25-29. Available from PMC: https://pubmed.ncbi.nlm.nih.gov/24881194/

20. Anderson SE, Whitaker RC. Household Routines and Obesity in US Preschool-Aged Children. J Amer Acad Pediatr [Internet]. 2010 March [cited 2020 Aug 25]; 125(3): 420-428. Available from: https://pediatrics.aappublications.org/content/pediatrics/125/3/4 20.full.pdf?ck=nck

21. Maddison R, Marsh S, Foley L, Epstein LH, Olds T, et al. Screen-Time Weight-loss Intervention Targeting Children at Home (SWITCH): a randomized controlled trial. Int J of Behav Nutr and Phys Act [Internet]. 2014 Sept [cited 2020 Aug 25]; 11 (111). Available from: https://ijbnpa.biomedcentral.com/ articles/10.1186/s12966-014-0111-2

22. Kothandan SK. School based interventions versus family based interventions in the treatment of childhood obesitya systematic review. Arc Pub Health [Internet]. 2014 January [cited 2020 Aug 25]; 72(3). Available from PMC: https://www.ncbi.nlm.nih.gov/pmc/articles/PMC3974185/

23. Brennan L, Walkley J, Wilks R. Parent- and AdolescentReported Barriers to Participation in an Adolescent Overweight and Obesity Program. Obes [Internet]. 2012 September [cited 2020 Aug 25]; 20(6); 1319-1324. Available from Wiley: https://onlinelibrary.wiley.com/doi/full/10.1038/oby.2011.358

24. Seburg EM, Olsen-Bullis BA, Bredeson DM, Hayes MG, Sherwood NE, et al. A Review of Pediatric Care-Based Childhood Obesity Prevention and Treatment Interventions. Curr Obes Rep [Internet]. 2015 June [cited 2020 Aug 25]; 4(2): 157-173. Available from PMC: https://www.ncbi.nlm.nih.gov/pmc/articles/PMC4512668/

25. Saelens BE, Sallis JF, Wilfley DE, Patrick K, Cella JA, et al. Behavioral weight control for overweight adolescents initiated in primary care. Obes res [Internet]. 2002 January [cited 2020 Aug 25]; 10(1): 22-32. Available from Wiley: https://onlinelibrary.wiley.com/doi/full/10.1038/oby.2002.4

26. Endevelt R, Elkayam O, Cohen R, Peled R, Tal-Pony L, et al. An Intensive Family Intervention Clinic for Reducing Childhood Obesity. J Amer Board Fam Med [Internet]. 2014 May [cited 2020 Aug 25]; 27(3): 321-328. Available from: https://www.researchgate.net/publication/262148884_An_ Intensive_Family_Intervention_Clinic_for_Reducing_Childhood_Obesity \#read

27. Uesugi KH, Dattilo AM, Black MM, Saavedra JM. Design of a Digital-Based, Multicomponent Nutrition Guidance System for Prevention of Early Childhood Obesity. J Obes [Internet]. 2016 August [cited 2020 Aug 25]. Available from PMC: https://www.ncbi.nlm.nih.gov/pmc/articles/PMC5007363/ 
28. Rizzo AS, Lange B, Suma EA, Bolas M. Virtual Reality and Interactive Digital Game Technology: New Tools to Address Obesity and Diabetes. J Diabetes Sci Technol [Internet]. 2011 March [cited 2020 Aug 25]; 5(2): 256-264. Available from PMC: https://www.ncbi.nlm.nih.gov/pmc/articles/PMC3125914/

29. Smith A. US Smartphone Use in 2015. Pew Research Center [Internet]. 2015 April [cited 2020 Nov 7]. Available from: https://www.pewresearch.org/internet/2015/04/01/ussmartphone-use-in-2015/

30. Thompson D, Cantu D, Ramirez B, Cullen KW, Baranowski T, et al. Texting to Increase Adolescent Physical Activity: Feasibility Assessment. Am J Health Behav [Internet]. 2017 July [cited 2020 Aug 25]; 40(4): 472-483. Available from PMC: https://www.ncbi.nlm.nih.gov/pmc/articles/PMC4922515/

31. Kowatsch T, Niben M, Shih I, Ruegger D, Volland D, et al. Text-based Healthcare Chatbots Supporting Patient and Health Professional Teams: Preliminary Results of a Randomized Controlled Trial on Childhood Obesity. Workshop, co-located with the 17th International Conference on Intelligent Virtual Agents (IVA 2017) [Internet]. 2017 August [cited 2020 Aug 25]. Available from: https://www.alexandria.unisg.ch/252944/2/2368_ Kowatsch\%20et\%20al\%202017\%20-

\%20THCB\%2OPEACH\%20Workshop.pdf

32. Jawed N, Mehndi A, Safdar N. Food Choices of Adolescents and Testing the Efficacy of Smart Phone Aided Nutrition Education Program (P21-062-19). Curr Dev Nutr [Internet]. 2019 June [cited 2020 Aug 25]; 3(2). Available from PMC: https://www.ncbi.nlm.nih.gov/pmc/articles/PMC6574381/

33. Partridge SR, Redfern J. Strategies to Engage Adolescents in Digital Health Interventions for Obesity Prevention and Management. Healthcare [Internet]. 2018 June [cited 2020 Aug 25]; 6(8): 70. Available from PMC: https://www.ncbi.nlm.nih.gov/pmc/articles/PMC6163226/ 